\title{
"Análisis del marketing cooperativo como estrategia en la internacionalización de las asociaciones"
}

\author{
"Analysis of cooperative marketing as a strategy in the internationalization of \\ associations"
}

\author{
Meyver Holguín Honores \\ Universidad Técnica de Machala \\ mholguin2@utmachala.edu.ec \\ Machala - Ecuador \\ María Cajo Torres \\ Universidad Técnica de Machala \\ mcajo1@utmachala.edu.ec \\ Machala - Ecuador

\section{Mabel Córdova Martínez} \\ Universidad Técnica de Machala \\ mcordova6@utmachala.edu.ec \\ Machala - Ecuador \\ Irene Sánchez-González \\ Universidad Técnica de Machala \\ isanchez@utmachala.edu.ec \\ Machala - Ecuador
}

\section{Resumen}

Determinar la efectividad del marketing cooperativo como herramienta para la internacionalización de las asociaciones. Se aplicó el método probabilístico con enfoque transversal a una muestra de 400 gestores de asociaciones de la provincia de El Oro. Se determinó que el conocimiento de marketing y la inclusión de estrategias de Marketing en las actividades de las asociaciones influye positivamente para lograr la internacionalización. Las asociaciones que tienen conocimiento de marketing e implementan estrategias de marketing cooperativo tienen resultados positivos en la internacionalización de sus productos.

Palabras claves: Internacionalización, Marketing cooperativo, Estrategias de marketing y marketing internacional. 


\begin{abstract}
Determine the effectiveness of cooperative marketing as a tool for the internationalization of associations. The probabilistic method with a cross-sectional approach was applied to a sample of 400 association managers from the province of El Oro. It was determined that the knowledge of marketing and the inclusion of Marketing strategies in the activities of the associations has a positive influence to achieve internationalization. Associations that have knowledge of marketing and implement cooperative marketing strategies have positive results in the internationalization of their products.
\end{abstract}

Keywords: Internationalization, Cooperative Marketing, Marketing Strategies and International Marketing

\title{
Introducción
}

El Marketing está dentro de los cuatro pilares de la gerencia, los cuales son la gestión humana, las finanzas, las operaciones y logística (Juárez y Barbosa, 2018). Por lo tanto, el marketing no puede ser solo la última etapa del proceso de operaciones comerciales de las asociaciones, esta manera de concebir el proceso de marketing simplificaría su función a simples ventas. (Dávalos, 2016) afirma que la cooperación del marketing dentro de las asociaciones son estrategias enfocadas en los objetivos de las mismas, su desempeño se efectúa dentro de un grupo de empresas con los mismos niveles de interés u objetivos. (Geldes y Heredia, 2016) afirma que, en casos específicos de innovación, la cooperación del marketing puede conducir a la llamada "innovación abierta", es decir, las empresas considerarán las ideas y capacidades tanto internas como externas al innovar, en lugar de tratar la "innovación cerrada" como un método más tradicional. Una empresa con conocimientos y capacidades propios ejecutará todo el proceso para lanzar nuevos productos y servicios innovadores hacia mercados extranjeros.

Por consiguiente, existen asociaciones que se concentran principalmente en las provincias de El Oro, Guayas y Los Ríos, las mismas que abarcan el 41\%, 34\% y 16\% de los productores. En la provincia de El Oro se sitúan la mayor parte de los pequeños productores del país (aproximadamente 42\%), mientras que los grandes productores principalmente en las provincias de Guayas y Los Ríos. Uno de los ejemplos más destacados de pequeños productores es la Asociación de Productores Bananeros "El Guabo", creada en 1997 es una asociación de pequeños productores de las provincias de Azuay, El Oro y Guayas. Hoy en día, cuenta con 350 miembros y emplea a más de 2.000 personas. (Ministerio del Comercio, 2017). ASOGUABO tiene como objetivo principal satisfacer las demandas de sus clientes con productos de alta calidad, bajo el sistema de comercio justo, con el objetivo de mejorar las condiciones de sus socios junto con su entorno social y laboral. (Ministerio del Comercio, 2017). Exporta exclusivamente con este sello Fairtrade a la Unión Europea, por usar este 
sello la Asociación recibe un premio por cada caja que es auditada periódicamente. Es más, Cuando el productor necesite la asistencia de un exportador para poder asumir las exportaciones Comercio Justo Fairtrade, el productor debe realizar una solicitud al certificador confirmando que necesita un exportador para exportar productos Comercio Justo Fairtrade en su nombre. De esta forma se dejará poco a poco a las multinacionales de EE. UU y se negociará directamente con la Unión Europea. (Palomeque, 2015)

Según estudios las estrategias del marketing cooperativo dentro de las asociaciones para su internacionalización se establecen mediante la siguiente estructura: Análisis del mercado extranjero por los cuales se establecen estudios sobre la demanda primaria, selectiva, y la segmentación. Su aplicación ayuda a determinar dónde están ubicados los posibles compradores potenciales del producto (Novillo, 2015). Un análisis de mercado permite a las asociaciones a determinar el segmento, las variables, categorización, formación de segmentos y conocer el perfil de cada segmento. (Serralvo y Tadeu, 2005). El segmento responde favorablemente a las cinco fuerzas de Porter: consumidores finales, zonas urbanas o rurales, ingresos medio-altos, beneficios, tipo de consumo de productos, cultura y compra a empresas que demuestran responsabilidad social (Novillo, 2015). En este punto también surge el mapa perceptual como una herramienta en la cual desarrolla trabajos para soportar el esfuerzo de posicionamiento de producto/marca. (Fernández, 2015) afirma que el mapa perceptual muestra de qué forma ve el consumidor la competencia en el mercado, y refleja la valoración que hace el mercado de un conjunto de marcas o productos señalan que el trazado de mapas perceptuales consiste en la elaboración de mapas que son representaciones visuales de la percepción del consumidor acerca de un producto, marca, compañía o cualquier otro objeto en dos o más dimensiones.

Las estrategias del marketing cooperativo basadas en comercio justo se dan mediante la identificación de las siguientes variables controlables en la que deben combinar la dirección de marketing para conseguir los objetivos previstos y se materializa en ocho instrumentos. (Martínez, Ruiz, y Escrivá, 2014). A nivel de producto, se analiza el grado de adaptación de los productos al mercado de destino que es medido en términos de diseño, calidad, servicio ofertado y servicio posventa. (Vallejo, 2016). A nivel de precio, se analiza el grado de adaptación de la estrategia de precios de las empresas al mercado de destino en adaptación a la demanda de los clientes internacionales. (Vallejo, 2016). A nivel de distribución, se analizaron los acuerdos de cooperación como modalidad estratégica para dirigirse a los mercados internacionales. A nivel de promoción, se analiza el número de actividades de promoción internacional. Las compañías deben comunicarse con los clientes actuales y potenciales, sin dejar al azar lo que desean comunicar. (Estrella, Jiménez, Ruiz, y Sánchez, 2014) 
El Marketing moderno a nivel de personas refleja parcialmente el marketing interno y el hecho que los empleados son parte fundamental para el éxito del marketing. A nivel de procesos refleja toda la creatividad, disciplina y estructura que se incorpora a la dirección de marketing. A nivel de programas refleja todas las actividades de la empresa que se dirigen al consumidor. (Villavicencio, Orellana, Pesantez, y Loor, 2015), afirma que las antiguas cuatro P's. A nivel performance se define de acuerdo con el marketing holístico, como las posibles medidas de resultados que tienen implicaciones financieras y no financieras (rentabilidad, así como capital de marca y de clientes), e implicaciones más allá de la empresa (responsabilidades social, legal, ética y comunitaria). La cooperación empresarial es un mecanismo a través del cual las organizaciones pueden unir su voluntad, recursos y planes para buscar metas comunes con el fin de ser más competitivas en el mercado internacional, de modo que puedan obtener diferencias estratégicas de inversión a través de productos. (Vélez, Beltrán, y López, 2019). Y el marketing cooperativo aporta con las siguientes ventajas a las asociaciones: Complementación de capacidades-Los participantes de la alianza no necesariamente son iguales. Por lo general tienen fortalezas y capacidades distintas y, en consecuencia, pueden complementarse y potenciarse. Para que una alianza tenga sentido la lógica debe ser dos son mejor que uno. (Pérez, 2020). Fuerza Negociadora-Sin duda se fortalece la capacidad de negociación frente a los proveedores (materias primas, insumos), suministradores de servicios (financieros, por ejemplo) y clientes (precios, términos de pago, plazos, etc.). (Pérez, 2020). Posibilidad de Marca-Posicionar una marca en un país y más aún en mercados internacionales supone normalmente un costo alto. Se requiere inversión en la mezcla promocional: publicidad, promoción de ventas, venta personal y relaciones públicas. Este costo puede ser distribuido entre los miembros de la alianza, permitiendo el posicionamiento de una marca propia que le da mayor sostenibilidad al negocio. (Paixao, Cunha, y Cruz, 2019). Menos Intermediarios-Aunque en muchos casos hay intermediarios que difícilmente podrán ser excluidos de la cadena, siempre existe la posibilidad de saltear uno o más eslabones respaldados en la alianza estratégica (mayor volumen). (Salvador, 2014) afirma que la internacionalización como un proceso de implicación creciente en operaciones internacionales. A lo largo de los años, la internacionalización ha sido un proceso de vital importancia para muchas empresas que han ampliado su segmento de mercado estas han visto una serie de ventajas que pueden ayudar a mejorar su posición competitiva en el mercado exterior. Además, la internacionalización de las asociaciones son procesos de cooperaciones estratégicas para el desarrollo diversificado de la geografía internacional, que desde una perspectiva de largo plazo es evolutiva y dinámica, y que gradualmente aporta a las diferentes actividades de la cadena valor y a la estructura organizativa de la empresa, con un compromiso e implicación creciente de sus recursos y capacidades con el entorno internacional, y basado en un conocimiento acumulativos. (Martínez, 2016) 
Los estímulos que motivan a las asociaciones a la internalización son los siguientes: La posibilidad de incrementar el número de clientes potenciales: La salida de las asociaciones empresariales a terceros mercados, repercutirá en la práctica con la elevación consecuente del número de clientes reales y potenciales. (Jerez y García, 2015). Crecimiento: La motivación principal para iniciar la actividad internacional es considerar dicha posibilidad como una estrategia básica para la búsqueda del crecimiento. La ponderación del crecimiento por la vía de la diversificación de mercados puede introducir además nuevas variables, como pueden ser la innovación y mayor flexibilidad en la gestión. (Jerez y García, 2015). Superación y actualización de la gestión empresarial: Para poder afrontar los nuevos restos derivados de la presencia en el exterior, que extrañan una necesaria mejora de gestión, a fin de superar con éxito las dificultades y potencialidades de la nueva situación internacionalizada de la asociación. (Delgadova y Gullerova, 2016). Diversificación del riesgo: Con la apertura de nuevos mercados se elimina para la asociación el riesgo de los desequilibrio y amenazas de operar en un único mercado sin posibilidad de alternativas. (Delgadova y Gullerova, 2016). Nueva imagen de marca: Las asociaciones internacionalizadas añaden a la cadena de valor una revalorización a su imagen de marca más dimensionadas y de multimercado. (Pedraza y Ramírez, 2015). Existencia de una demanda cada vez más global: Ante esta tendencia y el aprovechamiento de los nuevos sistemas cada vez más globales de distribución se facilitan los impulsos para la internacionalización. (Pedraza y Ramírez, 2015). Alcanzar mayor flexibilidad: Lo que se logra mediante una mejor posición competitiva a través de la expansión internacional (Pedraza y Ramírez, 2015).

\section{Materiales y Métodos}

La metodología utilizada en la presente investigación es de carácter cualitativo mediante la búsqueda de información secundaria en revistas, artículos científicos, blog, libros etc., sobre las asociaciones que ya ocupan mercados internacionales. (Herrera, 2017). Y de carácter cuantitativo: La investigación se aplicó a un universo conformado por 1200 asociaciones, pertenecientes a las industrias con mayor actividad (agricultura, acuicultura y pesca), de las cuales se depuraron 400 asociaciones que exportan y que no exportan sus productos, pero tienen el potencial para desarrollar este tipo de actividades al mercado internacional. Dentro de las 400 asociaciones se encuentran 1121 empresas de la Provincia de El Oro, las cuales fueron distribuidas en función a la cantidad de estudiantes de los horarios nocturno y matutino, 75 estudiantes en total. Se aplicó el método probabilístico con encuestas y escalas de Likert para la recolección de información, que se levantó desde diciembre del 2019 hasta febrero del 2020. Toda esta información ya obtenida y estudiada minuciosamente, se evidenciará en el desarrollo del presente proyecto. 


\section{Resultados y Discusión.}

La cooperación empresarial o alianza se crean por diferentes motivos estratégicos, instaurándose un cierto grado de interrelación entre las empresas participantes. (Fernández, 2015). A través de distintas formas estructurales, para mejorar en última instancia su posición competitiva al realizar el trabajo de forma más eficiente aprovechando las oportunidades que se presentan en un entorno competitivo. Esta investigación tuvo como propósito identificar y describir la importancia del marketing cooperativo para la internacionalización de las empresas, presentaremos los resultados del análisis de los datos obtenidos en nuestra investigación. Estos resultados mostrarán de manera general la importancia y la utilidad que tiene el marketing cooperativo, destacaremos especialmente las variables que han influido significativamente en la investigación, ofreciendo las posibles razones que han podido dar lugar a dichos resultados. Presentados en las siguientes figuras:

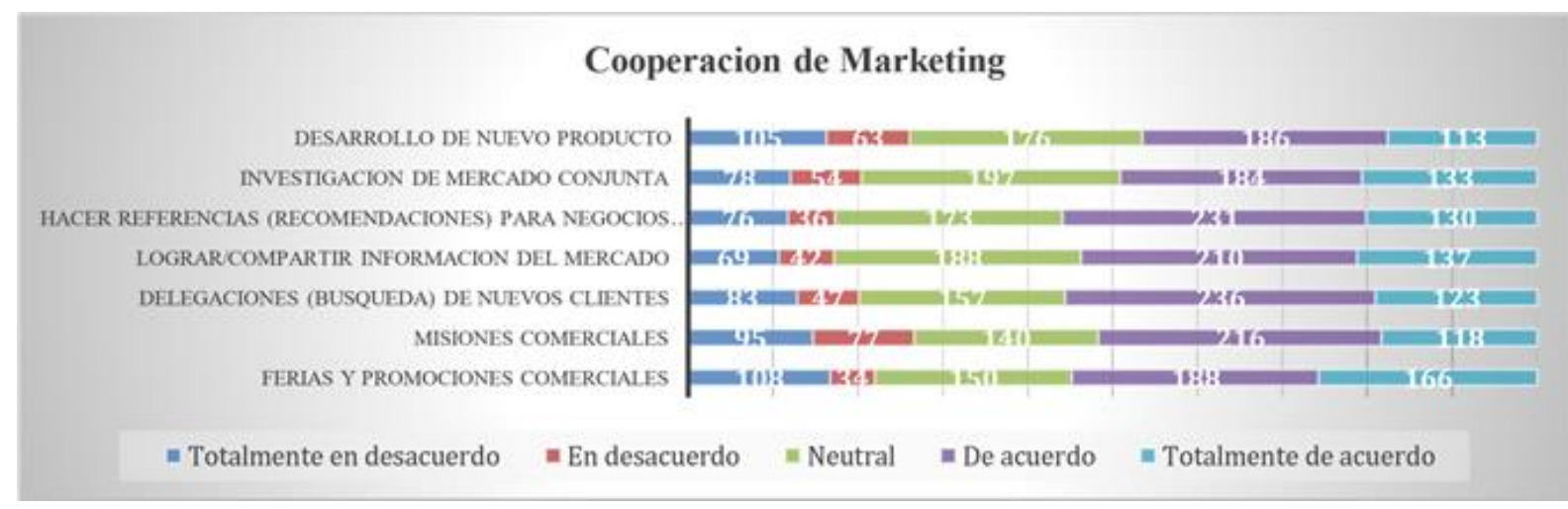

Figura 1 .

Cooperación de marketing Socios de las asociaciones de El Oro, muestra de 400 gestores de asociaciones donde se encuentran 1121 empresas.

\section{Intensidad de internacionalizacion.}

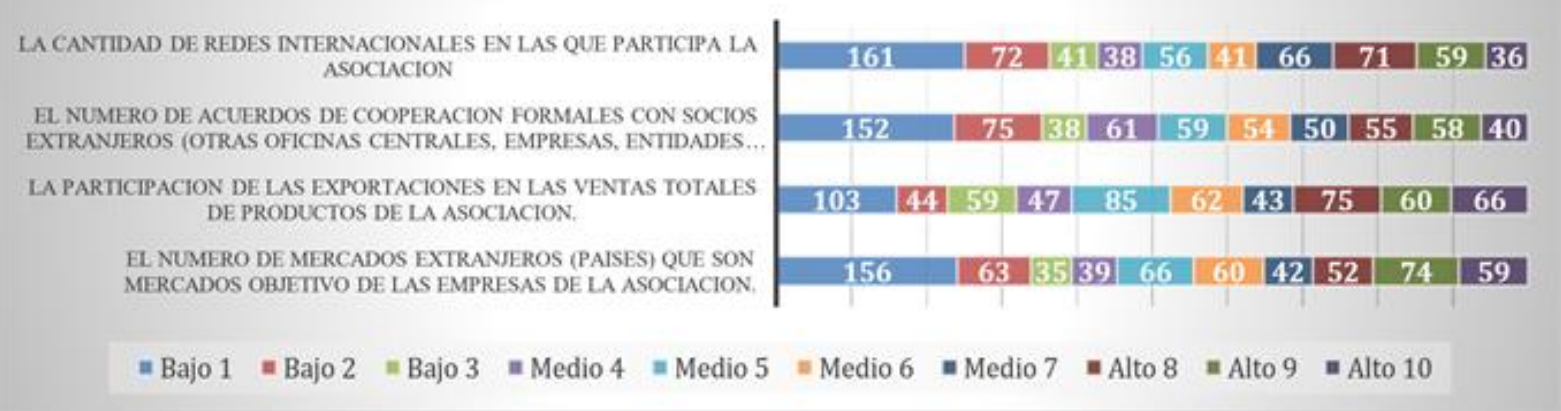

Figura 2.

Intensidad de internacionalización. Socios de las asociaciones de El Oro. 


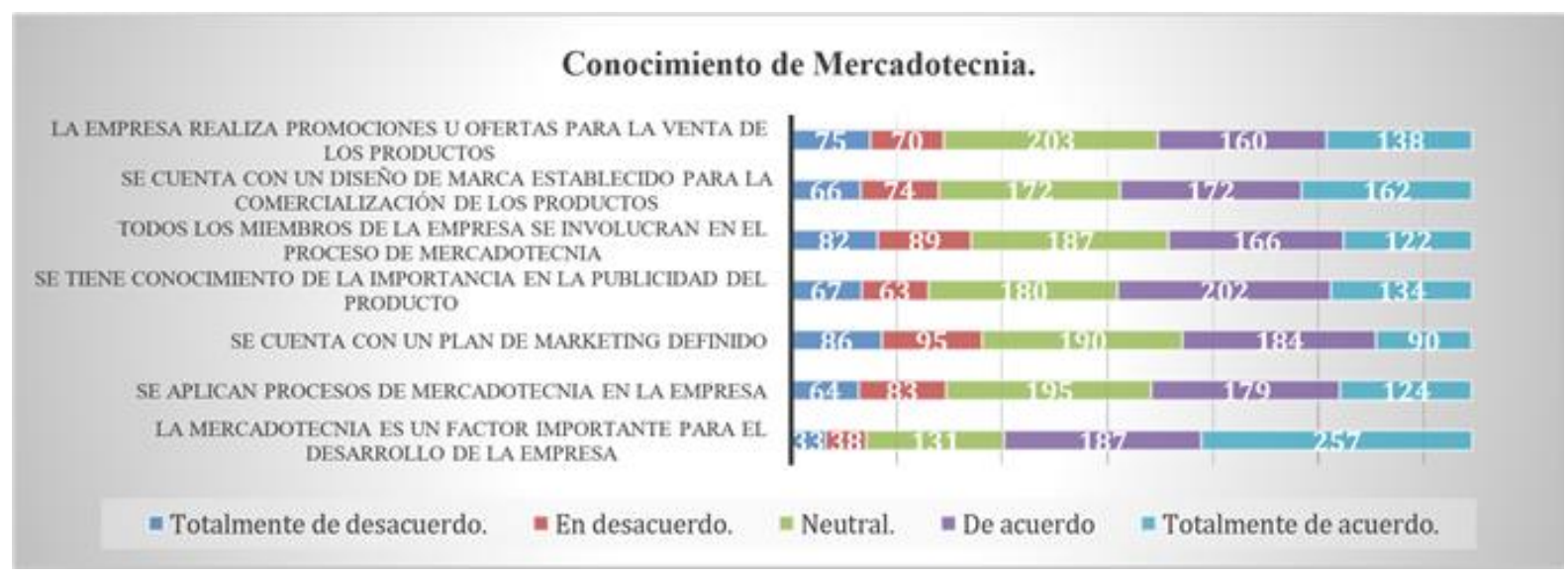

Figura 3.

Conocimiento de Mercadotecnia. Socios de las asociaciones de El Oro.

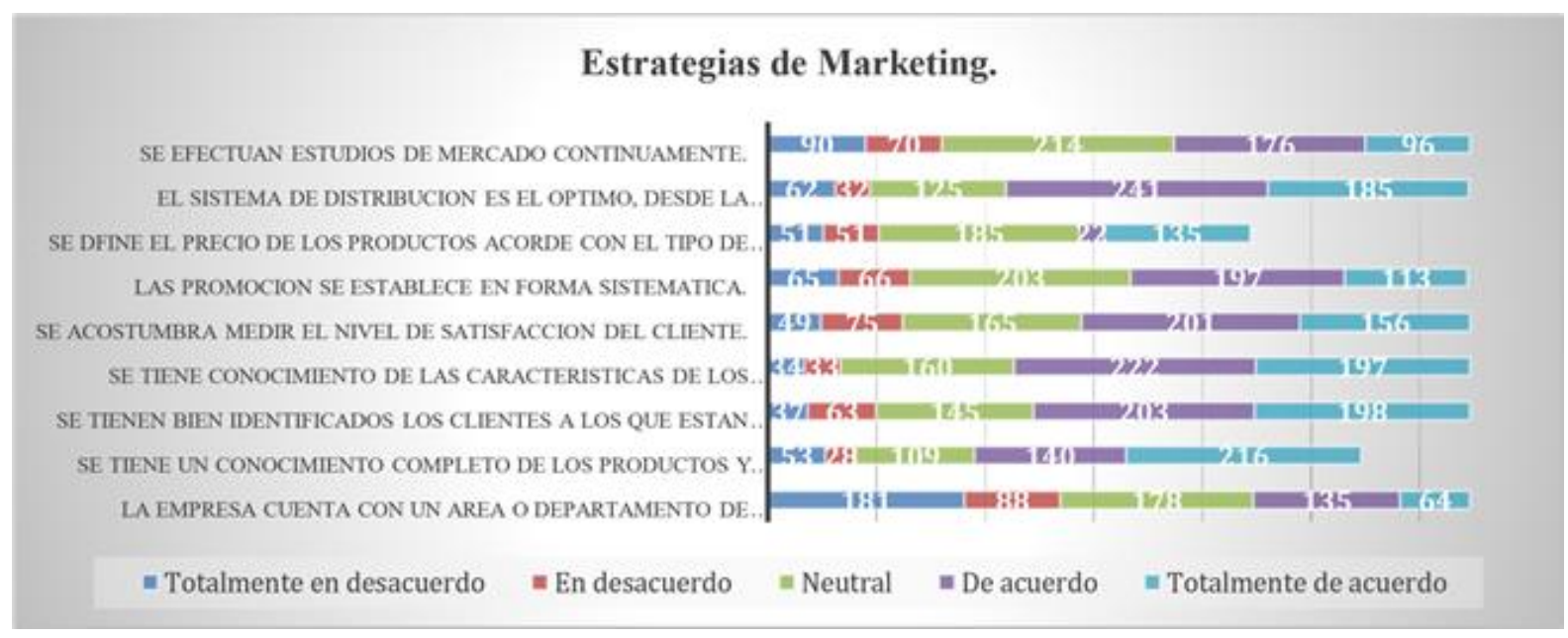

Figura 4.

Estrategias de marketing. Socios de las asociaciones de El Oro.

\section{Conclusiones}

Los resultados de investigación permiten responder a los objetivos propuestos, logrando concluir que para las asociaciones el marketing cooperativo es de mucha importancia ya que con esto lograran alcanzar a internacionalizar las empresas, es una herramienta efectiva, debido a que estas se unen a otras empresas para que así puedan llegar a otros mercados, optimizar recursos y obtener mejores promociones.

- En relación a la cooperación del marketing las asociaciones se mostraron de acuerdo en compartir información del mercado, ser partícipes en ferias y promociones comerciales que atraigan el interés del consumidor, así como buscar nuevos clientes, así como hacer una investigación de mercado conjunta que permita el desarrollo de nuevos productos. 
- Las asociaciones que exportan y aquellas que no, pero tienen la capacidad de exportar, con respecto al tema de intensidad de internacionalización, mostraron que existe un número bajo de mercados extranjeros que son mercado objetivo de la empresas de la asociación, por otro lado, mencionan que su participación en ventas tienen un número pequeño en el mercado exterior, así como el número de acuerdos de cooperación formales con socios extranjeros y la cantidad de redes internacionales en las que participa la Asociación son de intensidad baja.

- En lo que respecta al conocimiento sobre mercadotecnia, los asociados consideran que es un factor importante para el desarrollo de la empresa, en lo que respecta a la promoción de la venta de su producto tanto en el mercado nacional, como podría serlo en el internacional con la ayuda de las herramientas del marketing, sin embargo, no se cuenta con un plan de marketing definido en muchos casos.

- Estudiar el cambiante mercado en el que se encuentran, y así mismo contar con un departamento de marketing definido, puede ser un factor muy importante para que las ventas puedan lograr números mayores, por lo contrario, si se desconoce el mercado, no se cuenta con un departamento de marketing definido esto podría ser un motivo para que las ventas no alcancen volúmenes altos.

\section{Referencias}

Dávalos, E. (2016). Marketing Organizacional. Obtenido de http://repositorio.puce.edu.ec/handle/22000/10668

Delgadova, E., y Gullerova, M. (2016). The role of culture in International Marketing, International conference contemporary issues in theory and practice of management. 479, 57-60. Obtenido de https://doi. 10.1108 / 02651330810866290

Estrella, A., Jiménez, D., Ruiz, J., y Sánchez, M. (2014). ¿Cómo compiten las pymes en los mercados internacionales? Análisis de un clúster local con vocación exportadora. Academia Europea, 18(1), 87-99. Obtenido de https://n9.cl/i9drf

Fernández, E. (2015). “La cooperación empresarial”, introducción comercial española. España: Comercial española.

Geldes, C., y Heredia, J. (2016). Cooperación un tema pendiente para la innovación empresarial. 1(4). Obtenido de https://doi.org/10.11565/gesten.v1i4.26

Herrera, J. (2017). La investigación cualitativa. Obtenido de https://n9.cl/iw3lh 
Jerez, J., y García, A. (2015). La internacionalización de la empresa. Marketing internacional para expansión de la empresa. Madrid: ESIC Editorial. Obtenido de https://n9.cl/xnisd

Juárez, F., y Barbosa, D. (2018). Principios de marketing. Universal del Rosario, 10. Obtenido de https://doi.org/10.12804/ta9789587841183

Martínez, A., Ruiz, C., y Escrivá, J. (2014). Marketing en la actividad comercial. España: Mc Graw Hill Education. Obtenido de https://n9.cl/cai4

Martínez, J. (2016). El proceso estratégico de internacionalización de la empresa. teorías y propuestas para la dirección. Universidad de La Laguna. Obtenido de https://n9.cl/v931w

Ministerio del Comercio, E. (2017). Análisis del Sector Bananero Ecuatoriano. Produccion.gob.ec. Obtenido de https://n9.cl/banate

Novillo, G. (2015). Plan estratégico de marketing para la comercialización regional del excedente de banano orgánico y convencional. Una propuesta desde la visión del comercio justo en la provincia de El Oro. Editorial de las Fuerzas Armadas ESPE. Obtenido de https://repositorio.espe.edu.ec/handle/21000/12387

Paixao, D., Cunha, D., y Cruz, M. (2019). A study of the motivations for cooperating in the actions of the cvb of curitiba, state of paraná, among actors of the destination. Univali.br/períodicos, 355-379. Obtenido de http://dx.doi.org/10.14210/rtva.v22n2.

Palomeque, D. (2015). Análisis de la Variación de las Exportaciones de Banano de Ecuador hacia los Principales Socios Comerciales durante el periodo 2008 - 2013. Cuenca: Universidad de Azuay. Obtenido de https://n9.cl/g4dk

Pedraza, O., y Ramírez, M. (2015). Marketing Strategies for Hotel Industry Internationalization. ScienceDirect, 148, 271-279. Obtenido de https://doi.org/10.1016/j.sbspro.2014.07.043

Pérez, F. (2020). Asociatividad empresarial: estrategia para la competitividad de las PYMES en el Ecuador. Revista Eruditus, 1(2), 39-52. Obtenido de https://doi.org/10.35290/re.v1n2.2020.308

Salvador, A. (2014). La internacionalización mediante marketing colaborativo en redes sociales. Universidad de Almería. Obtenido de https://n9.cl/7w2wb

Serralvo, F., y Tadeu, M. (2005). Tipologías del posicionamiento de marcas. Un estudio conceptual en Brasil y en España Revista Galega de Economía. vol. 14(1-2), 115. Obtenido de https://n9.cl/74ssy. ISSN 1132-2799

Vallejo, L. (2016). Marketing en productos y servicios. Riobamba: La Caracola Editores. Obtenido de https://n9.cl/02fci

Vélez, S., Beltrán, J., y López, I. (2019). Business association and ambidiestro leadership as generators of innovation. Dialnet, 25(2), 51-72. Obtenido de https://n9.cl/id5o7

Villavicencio, D., Orellana, D., Pesantez, R., y Loor, A. (2015). The marketing tools and itc's: its use in smes for the business Development. Revista ECA Sinergia, 7. 